\title{
Trypanosomoses animales dans la Plaine de Mô au Togo
}

\author{
Essodina TALAKI ${ }^{1 *}$, Balabadi DAO ${ }^{2}$, Guiguigbaza-Kossigan DAYO ${ }^{3}$, \\ Essodong ALFA ${ }^{1}$ et Toï N'FEIDE ${ }^{1}$ \\ ${ }^{1}$ Université de Lomé (UL), Ecole Supérieure d'Agronomie (ESA), BP. 1515 Lomé, Togo. \\ ${ }^{2}$ Institut Togolais de Recherche Agronomique (ITRA), BP: 1163 Lomé, Togo. \\ ${ }^{3}$ Centre International de Recherche-Développement sur l'Elevage en zone Subhumide, \\ 01 BP : 454 Bobo-Dioulasso 01, Burkina Faso. \\ *Auteur correspondant, E-mail : talakiessodina@yahoo.fr, Tél : (228) 91681311
}

\section{RESUME}

Les trypanosomoses animales africaines (TAA) demeurent une des contraintes majeures au développement de l'élevage en Afrique subsaharienne. La présente étude a été conduite en mars 2013 dans cinq (5) localités correspondant aux cantons (Boulohou, Djarkpanga, Kangnigbara, Saiboudè et Tindjassi) de la Plaine de Mô située dans la Région Centrale au Togo. Des enquêtes transversales parasitologiques sur des bovins et entomologiques ont été menées. Ainsi, des prélèvements de sang à la veine jugulaire ont été effectués sur un effectif total de 449 bovins et des pièges à glossines ont été placés. Les résultats ont révélé la présence des trypanosomoses animales avec des prévalences comprises entre 32,56 et 43,59\% pour une moyenne de 37,64\%. Des infections à Trypanosoma vivax et Trypanosoma congolense ont été enregistrées avec une nette prédominance des infections à Trypanosoma vivax $(88,17 \%)$. La valeur moyenne des hématocrites des animaux est 23,4. Sur le plan entomologique, trois espèces de glossines ont été capturées: Glossina tachinoides avec une densité apparente par piège (DAP) de 0,$68 ; G$. palpalis palpalis avec une DAP de 0,52 et G. morsitans submorsitans avec un DAP de 0,01. De façon générale, l'ampleur des trypanosomoses animales dans la Plaine de Mô est très grande.

(C) 2014 International Formulae Group. All rights reserved.

Mots clés : Trypanosomoses, glossines, Plaine de Mô, Togo.

\section{INTRODUCTION}

L'Afrique sub-saharienne couvre une superficie d'environ 23 millions de $\mathrm{km}^{2}$ dont près de $40 \%$, soit 9,5 millions de $\mathrm{km}^{2}$ infestés par des glossines, insectes vecteurs des trypanosomoses humaine et animale (FAO, 1997). Selon les espèces animales attaquées, ces trypanosomoses prennent différentes dénominations: «Nagana» chez le bétail, «Dourine» chez les chevaux, «Surra» chez les chameaux, et «la maladie du sommeil» chez l'homme. Depuis plusieurs siècles, ces mouches tsétsé et les maladies transmises (trypanosomoses) constituent un problème majeur à la fois de santé et de développement agricole en Afrique subsaharienne (Peregrine, 1994 ; Swallow, 2000). Les incidences médicales et socio-économiques de ces trypanosomoses animales africaines transmises par les glossines sont très graves 
dans cette zone de l'Afrique. Les pertes directes sont représentées par la perte des animaux, la baisse de la productivité des animaux (viande et lait) et la perturbation de la reproduction (Chicoteau et al., 1990 ; Boly et al., 1991). Ces pertes sont estimées à 3 millions de têtes de bétail mort par an, une perte de $26 \%$ du rendement laitier et une réduction de $50 \%$ du nombre de troupeaux dans les zones à haute potentialité agricole (Hursey et Slingenbergh, 1997; Codjia et De La Rocque, 2001). Les mortalités ne sont pas trop fréquentes chez les races locales, alors qu'elles peuvent prendre des proportions importantes chez les races exotiques trypanosensibles. Quant aux pertes indirectes, elles sont nombreuses, passant par la limitation de l'introduction du bétail exotique plus performant mais trypanosensible, l'abandon des zones de pâturage infestées par les glossines, la réduction du rendement agricole des animaux, l'importation massive (viandes, produits laitiers et des trypanocides). $\mathrm{Au}$ Togo, en matière de trypanosomoses animales, depuis des travaux antérieurs réalisés à l'échelle nationale (Hendrickx et Napala, 1999), de récents travaux ont été entrepris dans l'objectif d'actualiser ces données afin de mieux définir des stratégies de lutte efficace contre ce fléau (Talaki et al., 2013a, 2013b, 2014). Par ailleurs, la Plaine de Mô, une des sous-préfectures de la région Centrale, en raison de son enclavement et de son isolement par des barrières naturelles (montagnes, rivières), a été pendant longtemps marginalisée et délaissée. Heureusement, depuis janvier 2012, l'État togolais a entrepris un vaste programme de développement de cette plaine à travers le Projet de Développement Rural Intégré de la Plaine de Mô (PDRI-Mô). Ce projet a pour but entre autres de lutter contre les maladies animales parmi lesquelles les trypanosomoses animales. C'est dans ce cadre que s'inscrit la présente étude avec pour objectif de connaître la situation épidémiologique des trypanosomoses bovines dans la Sous-Préfecture de la Plaine de Mô au Togo.

\section{MATERIEL ET METHODES Zone d'étude}

La zone d'étude correspond à la SousPréfecture de la Plaine de Mô. Elle relève administrativement de la région Centrale et est située dans la Préfecture de Sotouboua (Figure 1). Cette Sous-Préfecture est enclavée par les monts Fazao, faisant partie de la chaîne de l'Atakora qui prend le Togo en écharpe d'Est à l'Ouest. L'accès à la Plaine se fait exclusivement à partir de Bassar qui relève de la région de la Kara par une route non bitumée de $31 \mathrm{~km}$ de long de praticabilité permanente (ces deniers temps) à la suite des récents travaux pour le franchissement de la rivière Mô. Cette zone qui couvre une superficie de $1000 \mathrm{~km}^{2}$, est limitée au Nord par la rivière Mô, au Sud par les rivières Koué et Kpaza, à l'Est par les monts Fazao et à l'Ouest par le Ghana. Elle relève de l'étage climatique semihumide (pluviométrie moyenne de l'ordre de $1300 \mathrm{~mm} / \mathrm{an}$ ) de type soudano-guinéen perturbé par l'effet de l'harmattan (vent sec et poussiéreux du désert qui souffle du Nord au Sud) de décembre à février. Ce climat est marqué par deux grandes saisons : une saison pluvieuse d'avril à octobre et une saison sèche de novembre à mars. Le couvert végétal de la plaine est caractérisé par la prédominance d'une savane arborée moyennement claire, pouvant passer dans certaines zones du glacis de piedmont à des reliques de forêts sèches relativement denses à Pterocarpus erinaceus, Parkia biglobosa, Anogeissus sp, etc. La savane arborée, quant à elle, est peuplée principalement de Lophira alata, Terminalia macroptera, Pterocarpus erinaceus, Daniellia oliveri, etc. Les galeries forestières sont le plus souvent restreintes à quelques dizaines de mètres de largeur. La strate herbacée est essentiellement composée d'Andropogon sp, d'Hypparhenia sp, d'Aristida sp et de Loudetia sp. L'agriculture, l'élevage et le commerce constituent les principales activités économiques dans cette plaine. 


\section{Echantillonnage}

L'étude a été conduite en mars 2013 dans 05 cantons de la plaine de Mô: Djarkpanga, Boulohou, Kagnigbara, Saïboudè, et Tindjassé (Tableau 1). Au niveau de chaque canton, tous les troupeaux villageois bovins ont fait l'objet des prélèvements sanguin. Au total, 449 bovins ont été prélevés et répartis comme suit: Kangnigbara (30 bovins), Boulohou (50 bovins), Djarkpanga (129 bovins), Tindjassé (201 bovins) et Saïboudè (39 bovins).

\section{Enquête parasitologique}

Pour chaque animal de l'échantillon, du sang a été prélevé à la veine jugulaire dans un tube de prélèvement avec anticoagulant et conservé sous glace avant examen microscopique. A l'aide d'un laboratoire mobile utilisant comme source d'énergie un groupe électrogène, la mesure de l'hématocrite (Packed Cell Volume : PCV) et la recherche microscopique des trypanosomes vivants ont été réalisées sur place sur le terrain par la technique de Buffy Coat (Murray et al., 1977) dans un délai maximum de 4 heures. Les animaux parasitologiquement positifs ont été traités à l'acéturate de diminazène à la dose de $3,5 \mathrm{mg} / \mathrm{kg}$ de poids vif.

\section{Enquête entomologique}

L'enquête entomologique a été conduite dans la même période que l'enquête parasitologique afin d'identifier les espèces de glossines présentes, leur densité apparente par piège (DAP). Ainsi, pour chaque localité, en amont et en aval de la rivière environnante, des pièges biconiques standard (Challier et Laveissière, 1973) et monoconiques de type Vavoua (Laveissière et Grebaut, 1990) ont été placés de manière alternative et à intervalle d'environ $200 \mathrm{~m}$ pour une durée de 24 heures.

\section{Analyses statistiques}

Les données ont été saisies et analysées à l'aide du tableur Excel. La comparaison des valeurs moyennes a été effectuée à l'aide du test de $\mathrm{Chi}^{2}$ pour les prévalences et du test de l'écart réduit pour les hématocrites.

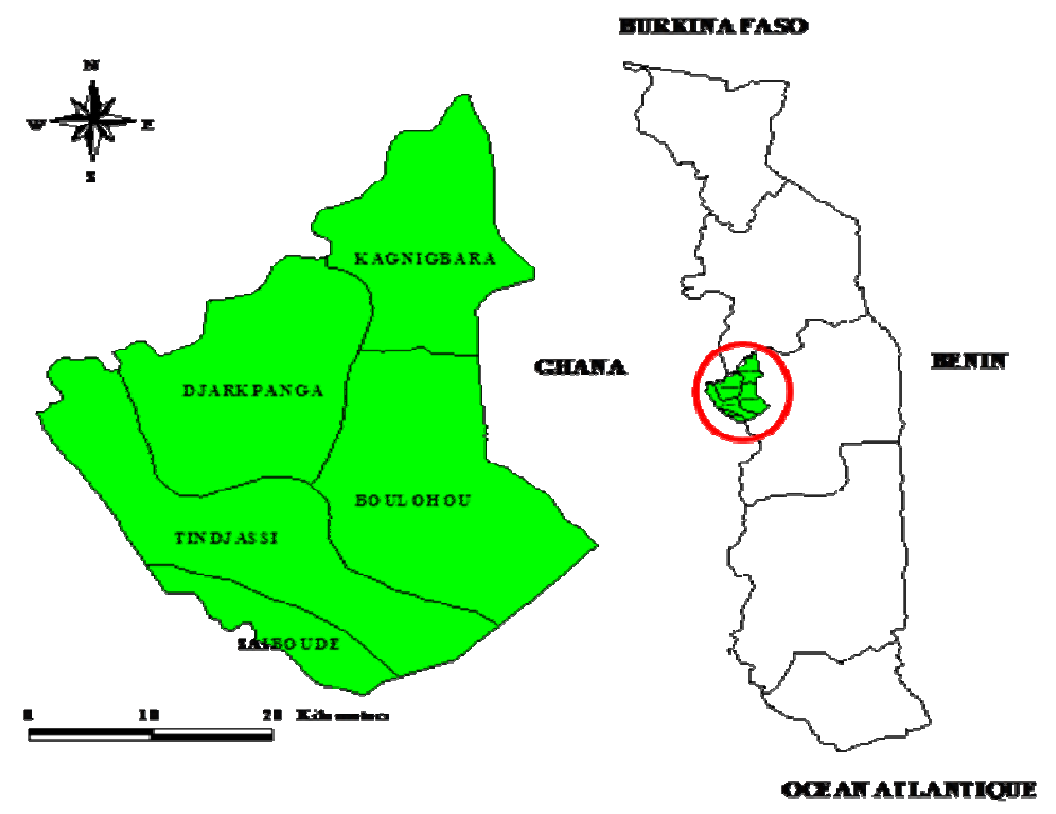

Figure 1 : Carte de la zone d'étude. 


\section{RESULTATS}

Prévalences parasitologiques et types d'infections

De manière générale, la maladie existe au niveau de la zone avec une prévalence parasitologique moyenne de $37,64 \%$. L'analyse des données au niveau cantonal a permis d'obtenir des valeurs comprises entre $32,56 \%$ et $43,59 \%$. Les plus fortes prévalences ont été obtenues à Saïboudè (43,59\%) suivi du canton de Bolohou (42\%) alors que la plus faible valeur des prévalences a été celle du canton de Djarkpanga (32,56\%). La Figure 2 représente les différentes valeurs des prévalences au niveau cantonal. La comparaison par le test de $\mathrm{Khi}^{2}$ de ces prévalences au niveau des cantons indique qu'il n'y a pas de différence significative (seuil 5\%). La Figure 3 présente les différents types d'infections ainsi que leur proportion. $\mathrm{Au}$ total, deux espèces de trypanosomes ont été identifiées. Il s'agit de Trypanosoma vivax et de $T$. congolense. La répartition de ces différents types d'infections montre une nette prédominance des infections à $T$. vivax $(88,17 \%)$. Les infections à $T$. congolens représentent $1,78 \%$ des cas enregistrés avec la possibilité d'infections mixtes $T$. vivax/T. congolense $(10,06 \%)$.

\section{Hématocrite des animaux}

Les différentes valeurs moyennes de l'hématocrite (PCV= Packed Cell Volume) des bovins sont consignées dans le Tableau 2. Pour l'ensemble de la zone étudiée, l'hématocrite moyen des animaux est $23,40 \pm$ 0,41 . Au niveau des cantons, l'hématocrite moyen des bovins se situe entre 22,60 $\pm 1,20$ (Boulohou) et 26,10 \pm 1,95 (Kagnibara). La comparaison des valeurs moyennes des hématocrites entre les différents cantons par le test d'écart réduit montre que la valeur moyenne de l'hématocrite des bovins à Kangnigbara est significativement supérieure (seuil 5\%) à celles des autres cantons (Boulohou, Djarkpanga, Tindjassé et Saïboudè). Au seuil 5\%, il n'existe pas de différence significative entre ces autres cantons. En d'autres termes, la valeur moyenne de l'hématocrite des bovins à Kagnibara est supérieure à celle des autres cantons.

Densités apparentes par piège des glossines $\mathrm{Du}$ point de vue entomomologique, trois espèces de glossines ont été identifiées dans la zone. Il s'agit de Glossina tachinoides, $G$. palpalis palpalis et $G$. morsitans submorsitans. Les espèces les plus importantes sont $G$. tachinoides et G. palpalis palpalis avec respectivement des densités apparentes par piège de 0,68 et 0,52 . S'agissant de l'espèce $G$. morsitans submorsitans, elle a été capturée uniquement dans le canton de Kagnibara avec une densité apparente par piège de 0,06 au niveau cantonal pour une densité apparente par piège de 0,01 pour l'ensemble de la zone étudiée. Les résultats entomologiques de ces enquêtes sont consignés dans le Tableau 3.

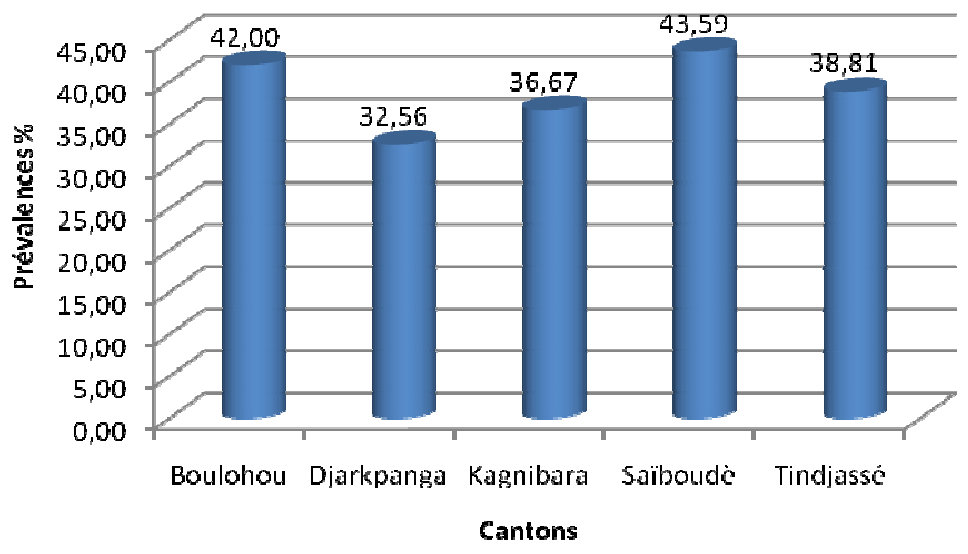

Figure 2 : Prévalences parasitologiques au niveau des cantons. 


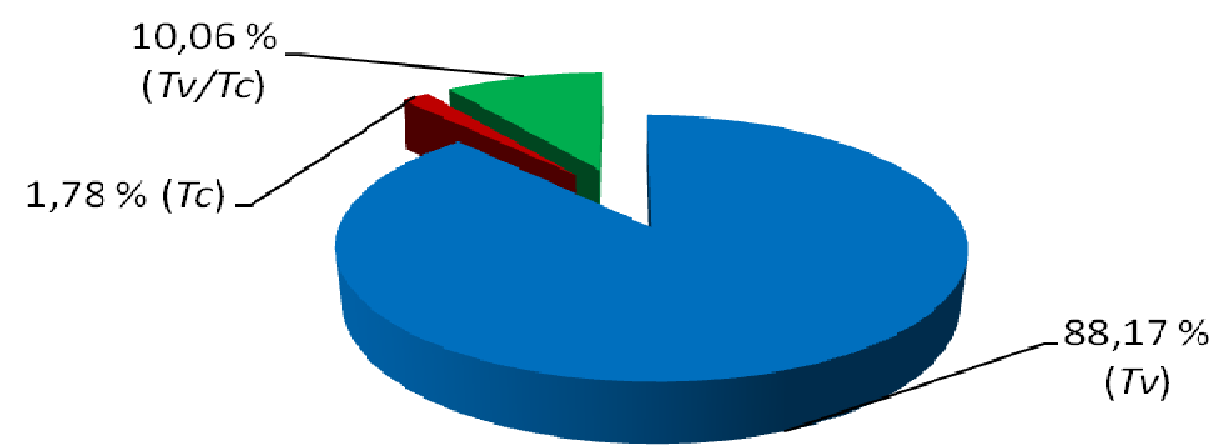

T.c : Trypanosoma congolense T.v : Trypanosoma vivax

Figure 3 : Proportion des différents types d'infections.

Tableau 1: Coordonnées géographiques des localités choisies.

\begin{tabular}{lccc}
\hline Cantons & Villages & Latitude & Longitude \\
\hline Boulohou & Boulohou & $8^{\circ} 46,298$ & $0^{\circ} 39,949$ \\
\hline \multirow{2}{*}{ Djarkpanga } & Kpangam & $8^{\circ} 49,169$ & $0^{\circ} 35,254$ \\
& Mampoula & $8^{\circ} 46,798$ & $0^{\circ} 29,647$ \\
\hline Kagnibara & Sangoule & $8^{\circ} 49,621$ & $0^{\circ} 34,559$ \\
\hline Saïboudè & Kagna & $8^{\circ} 50,082$ & $0^{\circ} 39,450$ \\
& Matchatom & $8^{\circ} 53,468$ & $0^{\circ} 36,014$ \\
\hline \multirow{3}{*}{ Tindjassé } & Saïboudè & $8^{\circ} 36,592$ & $0^{\circ} 28,528$ \\
& Ipouali & $8^{\circ} 45,115$ & $0^{\circ} 24,410$ \\
& Kandjo & $8^{\circ} 42,208$ & $0^{\circ} 26,814$ \\
& Nabou & $8^{\circ} 43,484$ & $0^{\circ} 23,545$ \\
& Taka & $8^{\circ} 46,576$ & $0^{\circ} 25,404$ \\
\hline
\end{tabular}

Tableau 2 : Hématocrite des bovins.

\begin{tabular}{lcccc}
\hline Cantons & Effectifs bovins & Hématocrite & Déviation standard & $\begin{array}{c}\text { Intervalle de confiance } \\
(\boldsymbol{\alpha}=\mathbf{0 , 0 5})\end{array}$ \\
\hline Boulohou & 50 & 22,60 & 4,34 & $22,60 \pm 1,20^{\mathrm{a}}$ \\
Djarkpanga & 129 & 22,99 & 4,25 & $22,99 \pm 0,73^{\mathrm{a}}$ \\
Kagnibara & 30 & 26,10 & 5,44 & $26,10 \pm 1,95^{\mathrm{b}}$ \\
Saïboudè & 39 & 23,38 & 4,80 & $23,38 \pm 1,51^{\mathrm{a}}$ \\
Tindjassé & 201 & 23,47 & 4,20 & $23,47 \pm 0,58^{\mathrm{a}}$ \\
Total général & 449 & 23,40 & 4,42 & $23,40 \pm 0,41$ \\
\hline
\end{tabular}


Tableau 3 : Densités apparentes par piège (DAP).

\begin{tabular}{lcccc}
\hline & \multicolumn{4}{c}{ Densités apparentes par piège (DAP) } \\
\hline Cantons & Nombre de piège & G.tachinoides & G. palpalis palpalis & G. morsitans submositans \\
\hline Djarkpanga & 15 & 0,40 & 0,13 & 0,00 \\
Tindjassi & 23 & 0,39 & 0,17 & 0,00 \\
Saïboudè & 15 & 0,60 & 0,60 & 0,00 \\
Kagnibara & 16 & 1,44 & 1,31 & 0,06 \\
Total & $\mathbf{6 9}$ & $\mathbf{0 , 6 8}$ & $\mathbf{0 , 5 2}$ & $\mathbf{0 , 0 1}$ \\
\hline
\end{tabular}

\section{DISCUSSION}

Les trypanosomoses animales sévissent dans la Plaine de Mô au Togo avec des prévalences moyennes (enregistrées au niveau des cantons) allant de 32,56 à 43,59\%. Pour l'ensemble de la zone étudiée, la prévalence parasitologique moyenne est de $37,64 \%$. Ces valeurs de prévalence enregistrées dans cette zone paraissent très élevées comparativement à la valeur moyenne nationale de 10\% (Dao et al., 2008) et aux prévalences enregistrées par Talaki et al. (2014) dans les régions des Savanes et de la Kara au Togo. En effet, Talaki et al. (2014), lors des enquêtes parasitologiques dans les régions des Savanes et de la Kara au Togo, avaient obtenu une plus forte prévalence de $21,56 \%$ dans les localités de Sarakawa et Tchitchao en octobre 2013. Cependant, cette prévalence moyenne de 37 , 64\% enregistrée dans la Plaine de Mô est comparativement proche des prévalences enregistrées par Talaki et al. (2013b) dans la préfecture de Vo au Togo, où une prévalence moyenne de $36,62 \%$ a été enregistrée. Cette relative forte prévalence parasitologique des trypanosomoses animales au niveau de la Plaine de Mô s'expliquerait par le fait qu'autrefois, la zone était presque enclavée, d'accès très difficile et ne bénéficiant pas des différentes campagnes de lutte entreprises dans le cadre des différents programmes nationaux. Aussi, elle est attenante au parc national Malfakassa-Faso qui aujourd'hui est vidé de sa riche faune sauvage victime d'un braconnage à outrance suite aux troubles socio-politiques qu'a connu le Togo durant la décennie 1990. Les glossines, ainsi privées de leurs hôtes sauvages, se seraient rabattues sur les bovins de la plaine. Par rapport à la méthode de diagnostic utilisée, elle est reconnue la plus pratique sur le terrain et permet d'identifier de façon rapide les animaux dont les parasitémies sont de l'ordre de 300 à 700 trypanosomes par $\mathrm{ml}$ de sang. Néanmoins, elle offre le désavantage de ne pas déceler les cas de faibles parasitémies (Murray et al., 1977; Very et al., 1990 ; Uilenberg, 1998; Desquesnes et De La Rocque, 1995). Des analyses complémentaires par la PCR avec les amorces spécifiques des parasites indiqueraient des prévalences plus élevées que celles décelées par la technique parasitologique classique par l'examen du buffy coat (Desquesnes et al., 1999 ).

Concernant les différents types d'infections, cette étude a révélé une nette prédominance des infections à $T$. vivax $(88,17 \%)$ contrairement aux études de Hendrichx et Napala (1999) qui avaient rapporté une très nette prédominance de l'espèce T. congolense (surtout en saison de pluies) au Nord du Togo. Par contre, cette prédominance des infections à $T$. vivax a été rapportée ces dernières années par Talaki et al. (2013a, 2013b, 2014) qui ont noté une nette prédominance de cette dernière espèce au Togo dans les régions de la Kara (56\%), dans la région des Savanes $(61,54 \%)$, dans la préfecture de Vô $(88,46 \%)$ et à la Station de Recherche d'Avétonou/ITRA (80,49\%). Cette forte prédominance des infections à $T$. vivax enregistrées s'expliquerait par les espèces de glossines présentes (glossines riveraines sont d'excellentes vectrices de trypanosomoses à $T$. vivax comparativement à $T$. congolense) mais aussi et surtout du rôle joué par les vecteurs mécaniques comme les tabanidés dans la transmission des infections à $T$. vivax (Dao et al., 2008). 
La valeur moyenne de l'hématocrite moyen des bovins est faible $(23,40 \pm 0,41)$, soit inférieure à 25 (seuil classiquement considéré comme révélateur d'un état anémique). Néanmoins, il est aussi important de préciser que bien que les trypanosomoses animales soient un facteur anémiant, le degré d'anémie chez un sujet dépend aussi bien de plusieurs autres facteurs tels que l'état nutritionnel, pathologique et de l'âge (Agyemang et al., 1990; Agyemang et al., 1991 ; Kaufmann et al., 1992).

Du point de vue entomologique, les glossines qui sont des insectes vecteurs de la maladie (trypanosomoses animales) ont été capturées et identifiées: G. palpalis palpalis, $G$. tachinoides et $G$. morsitans submorsitans avec une nette prédominance des deux premières espèces qui sont dites riveraines. Contrairement aux études réalisées par Talaki et al. (2014) dans les régions des Savanes et de la Kara, le piégeage a été relativement fructueux dans la présente étude puisque des densités apparentes par piège de 0,68 et 0,52 ont été respectivement enregistrées pour les espèces $G$. tachinoides et $G$. palpalis palpalis. En effet, ces derniers auteurs avaient, pour la région des Savanes et de la Kara, enregistré une densité apparente par piège de 0,01 pour l'espèce G. palpalis palpalis.

\section{Conclusion}

Les fortes prévalences enrégistrées dans la Plaine de Mô témoignent que les trypanosomoses animales constituent une importante contrainte pathologique dans cette zone. Une mise en place des stratégies adéquates de lutte contre ces maladies est alors indispensable pour la valorisation de cette zone du Togo.

\section{REMERCIEMENTS}

Les auteurs remercient:

- Le Ministère de l'Equipement Rural du Togo qui a financé cette étude à travers le Projet de Développement Rural Intégré de la Plaine de Mô (PDRI-MÔ) ;

- Les différentes institutions impliquées dans l'exécution de ce travail: 1'Institut Togolais de
Recherche Agronomique (ITRA) au Togo; l'Université de Lomé au Togo (UL) ;

- Les responsables et les agents impliqués (chauffeurs) de ces différentes structures ;

- Messieurs TSINI K. Agbéviadé, ISSIFA Soulé, BAKOSSI Oumborbé pour la collecte des données sur le terrain.

\section{RÉFÉRENCES}

Agyemang K, Dwinger RH, Little DA, Leperre P, Grieve AS. 1991. Interaction between physiological status in N'dama cows and trypanosome infections and its effect on health and productivity of cattle in Gambia. Acta Tropica, 50: 91-99.

Agyemang K, Dwinger RH, Touray BN, Jeannin P, Fofana D, Grieve AS. 1990. Effects of nutrition on degree of anaemia and live weight in N'Dama cattle infected with trypanosomes. Livestock Production Science, 26: 39-51.

Boly H, Thombiano D, Humblot P, Thibier M. 1991. Influence de Trypanosoma congolense sur la fonction sexuelle de taurins Baoulé. Revue d'Elevage et de Médecine Vétérinaire des Pays Tropicaux, 44: 475-480.

Challier A, Laveissière C. 1973. Un nouveau piège pour la capture des glossines (Glossina : Diptera, Muscidae) : description et essai sur le terrain. Cahiers ORSTOM, série Entmologie Médicale et Parasitologie, 10(4): 251-262.

Chicoteau P, Bassinga A, Sidibe I, Pobel T, Richard X, Clausen P. 1990. Influence de l'exposition à un risque trypanosomien élevé sur la reproduction des vaches Baoulé au Burkina Faso. Revue d'Elevage et de Médecine Vétérinaire des Pays Tropicaux, 43(4): 473-477.

Codjia V, De La Rocque S. 2001. Programme de lutte contre la trypanosomose africaine (PLTA). In: Utilisation des trypanocides en Afrique subsaharienne. Actes du séminaire sous-régional. Dakar, EISMV, du 06 au 09 février 2001, 45-46.

Dao B, Sidibé I, Hendrickx G, Belem AMG, De La Rocque S. 2008. Impact de la sécheresse et la dégradation des aires 
protégées sur la répartition des trypanosomiases bovines et de leurs vecteurs dans le bassin versant de l'Oti au Nord du Togo. Revue d'Elevage et de Médecine Vétérinaire des Pays Tropicaux, 61(34): 153-160.

Desquesnes M, De La Rocque S. 1995. Comparaison de la sensibilité du test de Woo et d'un test de détection des antigènes de Trypanosoma vivax chez les moutons expérimentalement infectés avec une souche guyanaise du parasite. Revue d'Elevage et de Médecine Vétérinaire des Pays Tropicaux, 48 : 247-253.

Desquesnes M, Michel JF, De La Rocque S, Solano P, Millogo L, Bengaly Z, Sidibé I. 1999. Enquête parasitologique et sérologique (Elisa-indirect) sur les trypanosomes bovins dans la zone de Sidéradougou, Burkina Faso. Revue d'Elevage et de Médecine Vétérinaire des Pays Tropicaux, 52(3-4) : 223-232.

FAO. 1997. Production Yearbook. FAO: Rome; 239.

Kaufmann J, Dwinger RH, Hallebeek A, Van Dijk B, Pfister K. 1992. The interaction of Trypanosoma congolense and Haemonchus contortus infections in trypanotolerant N'dama cattle. Veterinary Parasitology, 43: 157-170.

Laveissière C, Grebaut P. 1990. Recherche sur les pièges à glossine (Diptera : Glossinidae). Mise au point d'un modèle économique: le piège «Vavoua». Tropical Medecine and Parasitology, 41 : 185-192.

Hendrickx G, Napala A. 1999. Le contrôle de la trypanosomose «à la carte»: une approche intégrée basée sur un Système d'Information Géographique. Académie Royale des Sciences d'Outre-Mer, Classe des Sciences naturelles et médicales, Mémoire in- $8^{\circ}$, Nouvelle Série, Tome 24, fasc. 4, Bruxelles.

Hursey BS, Slingenbergh J. 1997. The tsetse fly and its effects on agriculture in sub-
Saharan Africa. Revue Mondiale de Zootechnie, 84/85: 67-73.

Murray M, Murray PK, Mc Intyre WIM. 1977. An improved parasitological technique for the diagnosis of African trypanosomiasis. Transactions of Royal Society of Tropical Medecine and Hygene, 71: 325-326.

Peregrine AS. 1994. Chemotherapy and delivery systems: haemoparasites. Veterinary Parasitology, 54: 223-248.

Swallow BM. 2000. Impacts of Trypanosomiasis on African Agriculture. PAAT Technical and Scientific Series 2. FAO: Rome, Italy; 52.

Talaki E, Dao B, Kulo A, N'Féidé T. 2013a. Enquêtes entomologique et parasitologique sur les trypanosomoses bovines à la Station d'Avétonou/ITRA, au Togo. Bulletin of Animal Health and Production in Africa, 61(1): 37-42.

Talaki E, Dao B, Dayo G-K, N'féidé T, Sodji K. 2013b. Etudes entomologiques et parasitologiques des trypanosomoses bovines dans la préfecture de Vo au Sud du Togo. Revue Africaine de Santé et de Productions Animales (RASPA), (Sous presse).

Talaki E, Dayo G-K, Akoda K, Dao B, Passaw Babi P, Alfa E, Lombo Y, Boukaya G, Tchamdja E, Kpabéba AD. 2014. Epidemiology of bovines trypanosomosis in Savannah and Kara regions in Northern Togo. International Journal of Agricultural and Soil Science (IJASS), 2(8): 126-131.

Uilenberg G. 1998. A Field Guide for the Diagnosis, Treatment and Prevention of African Animal Trypanosomosis. FAO: Rome, Italie; 158.

Very P, Bocquentin R, Duvallet G. 1990. Sensibilité de la double microcentrifugation pour la recherche des trypanosomes. Revue d'Elevage et de Médecine Vétérinaire des Pays Tropicaux, 43: 325-329. 